\title{
IR reflectivity measurements depending on carbon film thickness
}

\author{
C. Desgranges, C. Balorin, J. Bucalossi, D. Garnier, D. Guilhem, P. \\ Messina
}

\author{
Département de Recherche sur la Fusion Contrôlée \\ cea Cadarache \\ 13108 Saint Paul lez Durance
}

Abstract: In thermonuclear controlled fusion machines using magnetic confinement, carbonisations are realised to prevent metal impurities to enter into the fusion plasma made with hydrogen elements; it consists in helium glows in which methane gas is injected. The methane molecule is broken and the carbon deposits on all inside vessel surfaces : inner walls as well as optic elements like windows and mirrors. We studied the dependence of the reflectivity of infrared thermography stainless steel mirrors with carbon films thickness in the 3-5 $\mu \mathrm{m}$ bandwidth. The presented results show a decrease of less than $10 \%$ of the temperature announced by the camera .

\section{Magnetic fusion:}

Thermonuclear controlled fusion [1] research is made in the aim to reproduce solar energy in a controlled way. Fusion principle is to move closer two hydrogen atoms so that they form an heavier atom meanwhile an energetic neutron is released. The energy of this neutron will be recuperated to produce electricity. To bring closer hydrogen atoms, two conditons must be realised. First, confinement is needed. It is obtained in our case with magnetic strength instead of gravity as in stars ; another way is to use inertial confinement [1] where laser or particles beams compress hydrogen atoms. To keep particles magnetically trapped, magnetic lines are closed on themselves forming a torus shape. This is a tokamak machine [fig 1] .

Second, a high temperature is needed to overcome repulsion strength $\left(>10^{\circ} \mathrm{C}\right)$; at these temperatures, atoms are separated in ions and electrons : they form a plasma which is globally neutral. To reach such high temperatures, additional heatings are used based on neutral particle beams and high frequency waves such as ion cyclotron, lower hybrid and electron cyclotron .

Although plasma particles are trapped in non-material magnetic barriers, atomic collisions appear and diffusion, convection,conduction and radiation phenomena occur; this implies heating of contact surfaces. That is why an infrared thermographic system is developped to measure and survey these heatings which can lead to a failure of one of the plasma facing component inside the tokamak [2] .

\section{IR thermographic diagnostic:}

The Tore Supra IR thermographic diagnostic consists, until now, in three endoscopes [fig 2] situated at $120^{\circ}$ one from the other on the top of the tokamak; they are remotely controled so that every element inside the vessel can be observed .

Images of objects inside the vessel are captured by a movable stainless steel mirror and returned to an optical system through a sapphire window. This window is necessary to keep 
ultra high vacuum inside the torus vessel. Inframetrics 3-5 $\mu \mathrm{m}$ cameras recuperate images from the optical system which transported them .

Plasma inside the tokamak encounters surfaces made of, or covered with graphite. This graphite is sputtered and redeposited during or after plasma is stopped. Furthermore carbonisations are made to cover metal inner walls of the vessel with carbon so that metal impurities rate inside the plasma is lower. Carbonisation, and its erosion, was extensively studied $[3,4,5]$ but to our knowledge no measure on its effect on infrared mirror reflectivity was carried out. So did we. We deposited carbon layers on our stainless steel mirrors to determine from which thickness mirror reflection is affected.

\section{3 . Experimental apparatus :}

Our carbonisation system was installed in a special vessel [fig 3]. To realise carbonisation $\mathrm{He}$ or $\mathrm{D}_{2}$ or $\mathrm{H}_{2}$ glow plasmas can be used in which few per cent of $\mathrm{CH}_{4}$ or $\mathrm{CD}_{4}$ are in addition . Methane molecule is broken by collisions with plasma particles. Carbon atoms deposit on all surfaces . In our case, the plasma was made with helium and methane gas was injected .

Two $\lambda / 2$ polished mirrors, set back to back, are supposed to be hidden from carbon deposition and centimeter by centimeter are exposed to the glow (see fig 3). The deposited layer thickness is indicated by a microbalance diagnostic. The following parameters were established for carbonisation :

$\mathrm{P}_{\mathrm{CH} 4} / \mathrm{P}_{\mathrm{He}}=0,065$

total pressure $(\mathrm{He}+\mathrm{CH} 4): \mathrm{P}_{\text {tot }}=1,15 \mathrm{~Pa}$

plasma current density : $\mathrm{j}=12 \mu \mathrm{A} / \mathrm{cm} 2$

carbon deposit speed : $\mathrm{f}_{\mathrm{C}}=1$ monolayer in $10 \mathrm{~s}$

vessel temperature : $\mathrm{T}_{\text {deposit }}=150^{\circ} \mathrm{C}$

The total deposit duration was less than two hours .

\section{4 . Experimental results :}

Six carbon films $1 \mathrm{~cm} \times 3 \mathrm{~cm}$ wide were obtained : 50 monolayers, 150, 250, 350, 450 and 550 monolayers thick [ fig 4] following the microbalance indication .

The two mirrors present the same visual aspect. A correlation between film thickness and colour was established by J. Winter in his study of carbonisation in Textor tokamak [5] . Using this correlation, we find a good agreement between thickness given by the microbalance and the colour of films deposited on our mirrors .

Reflectivity results for both mirrors are presented below [fig 5]. Reflectivity is constant from 0 to 150 then it decreases from 150 monolayers deposited; as expected the thickest film has the worst reflectivity. We compare the reflectivity of mirrors just beeing polished before deposition and what we call the 0 monolayer situated in a place always under the cache. There is a minor difference between the two which is inside error bars .

Reproducibility of our reflectivity measurements is in the order of $+/-2 \%$.

Our deposits need special care because of their poor adhesion : we lost part of the thickest deposits by rubbing with a plastic bag. Mirrors surfaces were made very smooth and then it is not so surprising that our films have little adhesion as thick they are . Furthermore, electrostatic interaction may increase this effect. Indeed carbonisation films have quite high electrical resistivity . For example, on Textor samples $1-10^{6} \Omega \mathrm{cm}$ was typically measured [5] .

We observed too that the two films 450 and 550 monolayers show waves. These waves are more important with the increase of thickness. J. Winter observed and measured the same phenomenon [5]. This author says this appears during air exposure. We are not able to 
confirm this because there was no window in our carbonisation vessel where we could see mirrors still under vacuum before air exposure [fig 6] .

Measurement of the 550 monolayers zone where the deposit is and where it peeled, was undertaken . Reflectivity of the undamaged part of the film is the lowest. Reflectivity of the peeled film is half between that latter and that of $0-150$ monolayers .

If we consider the worst result which means 550 undamaged monolayers deposited, we find that the real temperature is underestimated by less than 10 per cent; this may be dangerous in our application especially at high temperatures : for example, the copper melting point is at $1083^{\circ} \mathrm{C}$, with our results the temperature indicated by infrared cameras is around $975^{\circ} \mathrm{C}$, but inside the copper 15 bar water circulate to refregirate the plasma facing components which means that with our measurement we wouldn't understand why there is a water leak in the machine; this leak implies no experiment for three months to mend damaged parts and to restart the machine.

\section{5 . Conclusion :}

We realised carbonisation with variable thicknessses on two polished stainless steel mirrors used in the infrared 3-5 $\mu \mathrm{m}$ band. Their reflectivity before and after carbonisation was measured, on the different thicknesses of the films formed. Below 150 monolayers the reflectivity does not change. Beyond 150 monolayers, a decrease is observed. If deposit peels, reflectivity is only modified but does not come back to the value with few deposit . Over 450 monolayers the deposit shows waves which show poor film adhesion. The two mirrors have the same results even if they were set back to back for carbonisation, one looking at the anode, the other looking at the opposite. We deduced from these reflectivity measurements that in the case of the thickest deposit the real temperature of the tokamak element is under evaluated by less than $10 \%$.

Acknowledgements : The authors want to thank A Grosman for fruitfull discussion and P. Maillet and his team for their advices and help for that work to be done .

\section{References :}

[1] Commissariat à l'Energie Atomique, La fusion thermonucléaire contrôlée par confinement magnétique , Masson, 1987

J. Adam, La fusion nucléaire une source d'énergie pour l'avenir?, Pour la Science Diffusion Belin, 1993

[2] D. Guilhem, QIRT 92, 7-9 july 1992, eurotherm seminar 27

[3] A. Grosman, private communication

[4] E. Gauthier, P.H.D. thesis, Universite de Provence Aix-Marseille I, 1989

[5] J. Winter, Journal of Nuclear Materials, 161, 1989, p265

\section{Captions :}


http://dx.doi.org/10.21611/qirt.2000.049

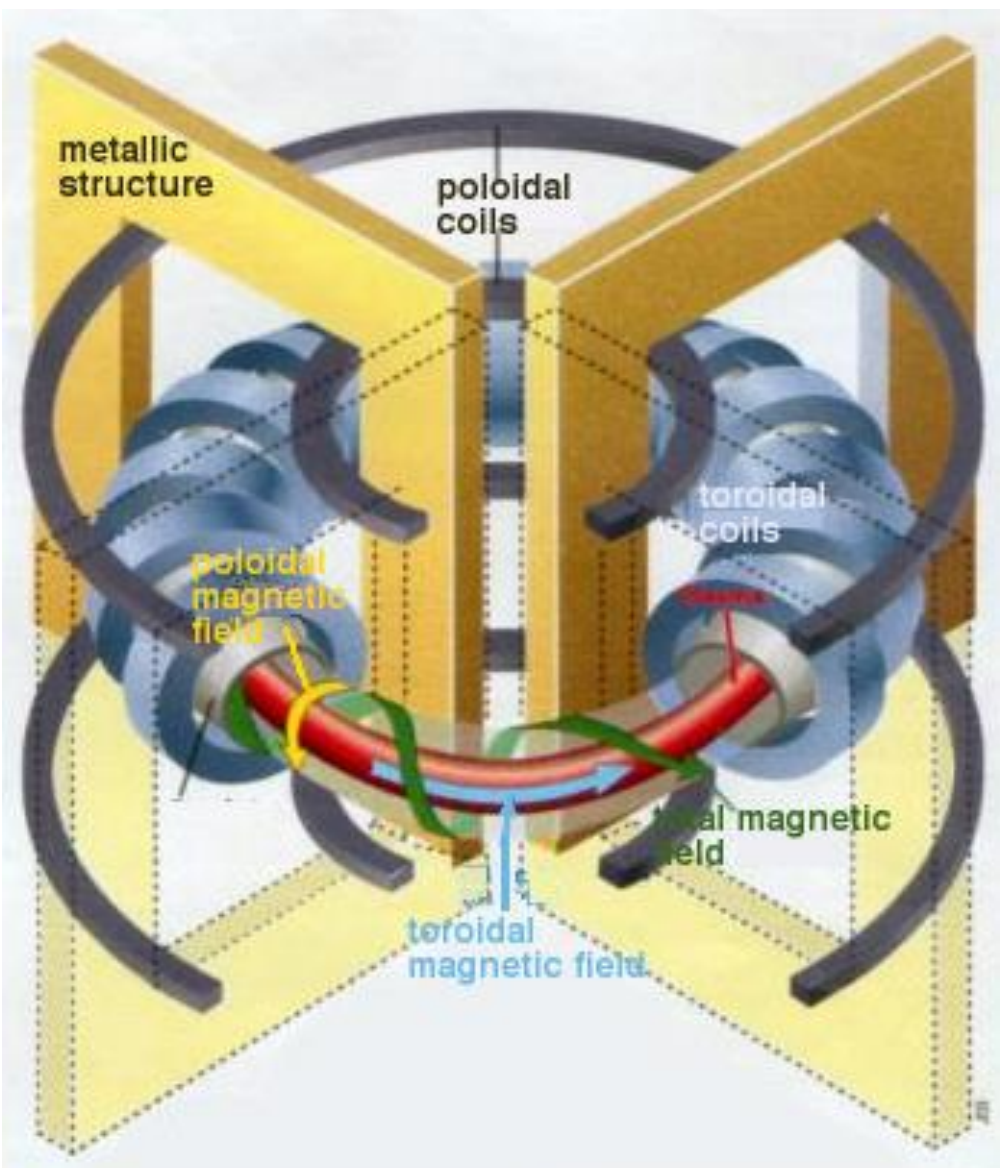

fig.1 : Tokamak principle

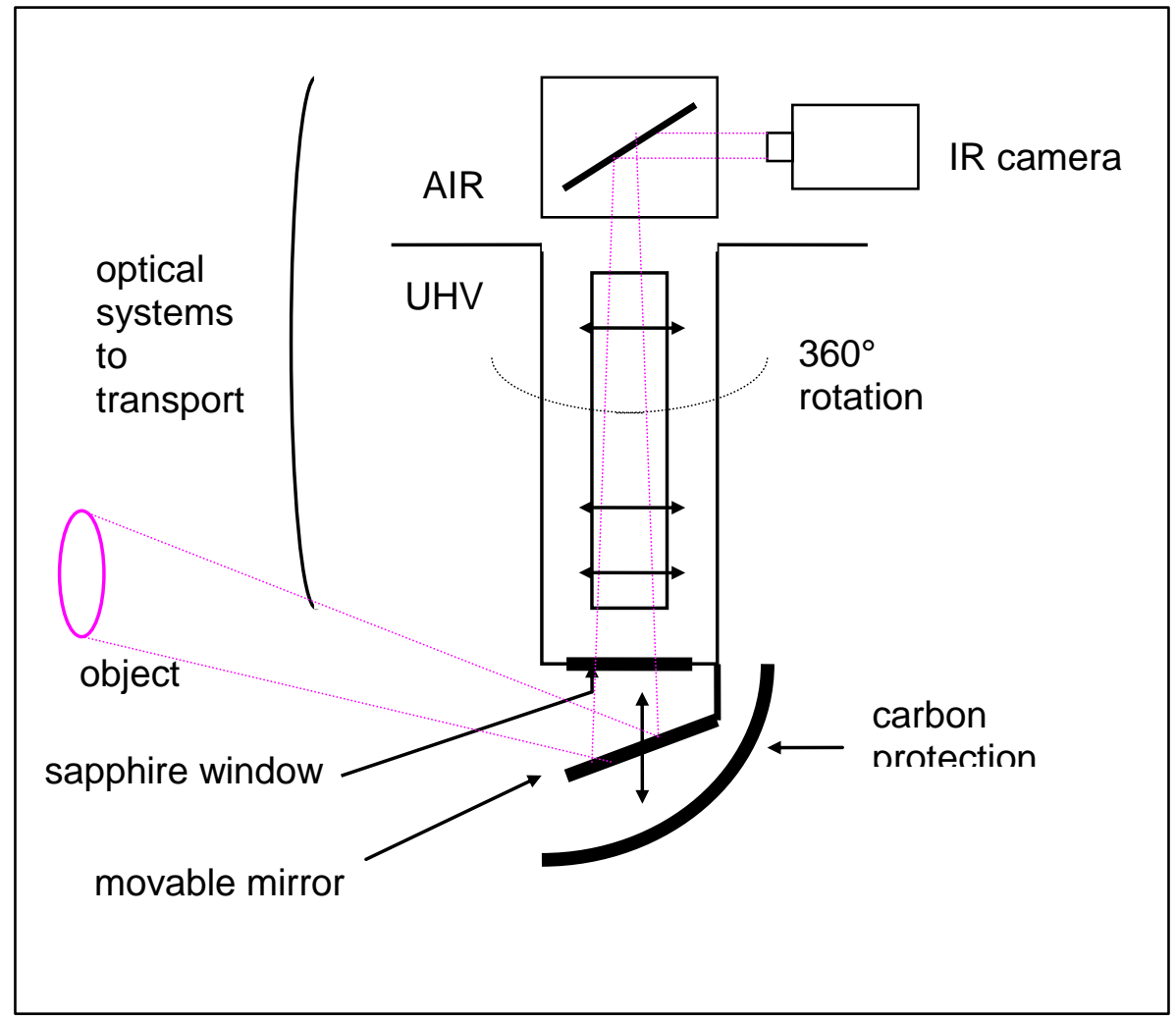


fig.2 : endoscope principle

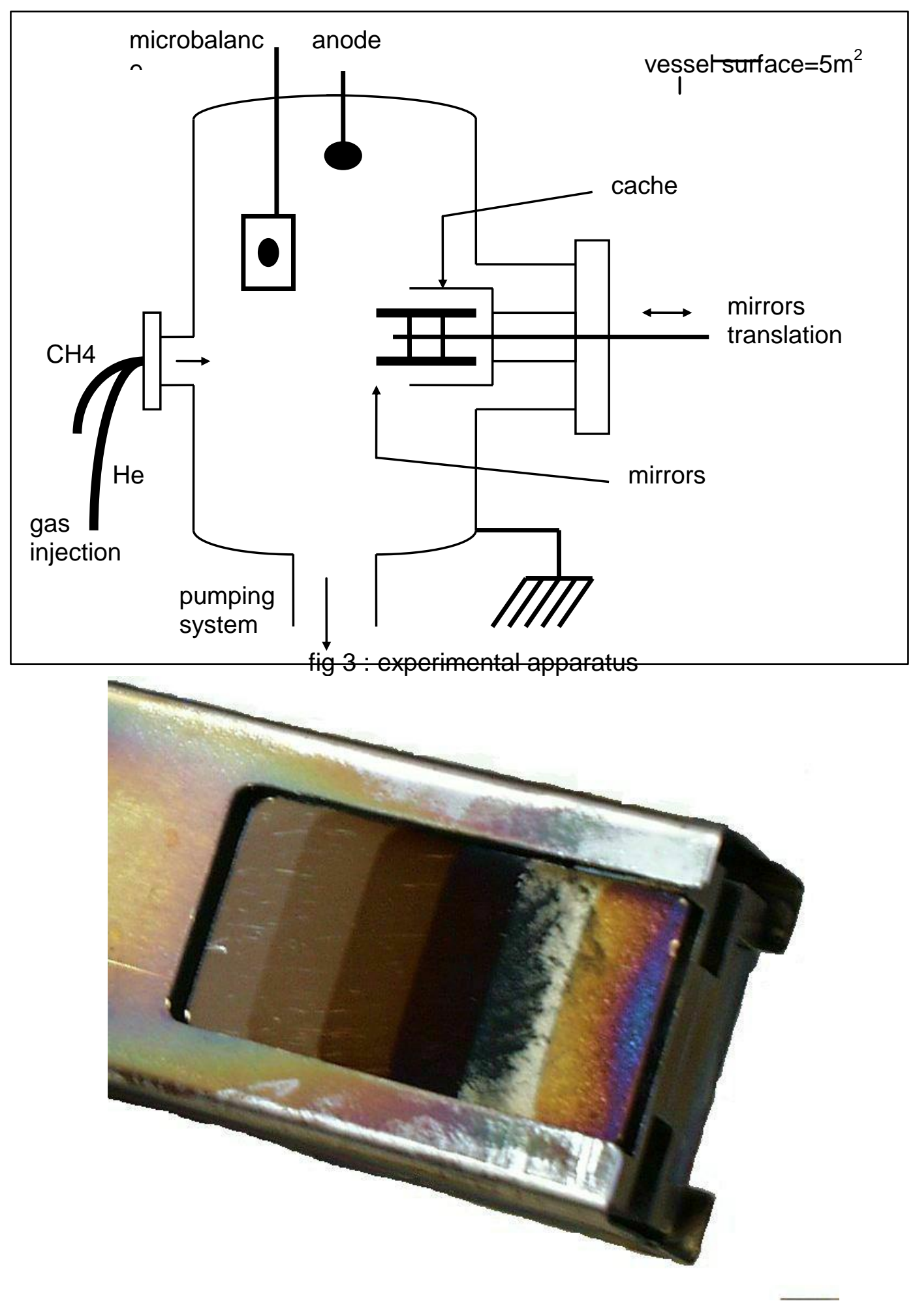

fig 4 : stainless steel mirror after carbon deposition 


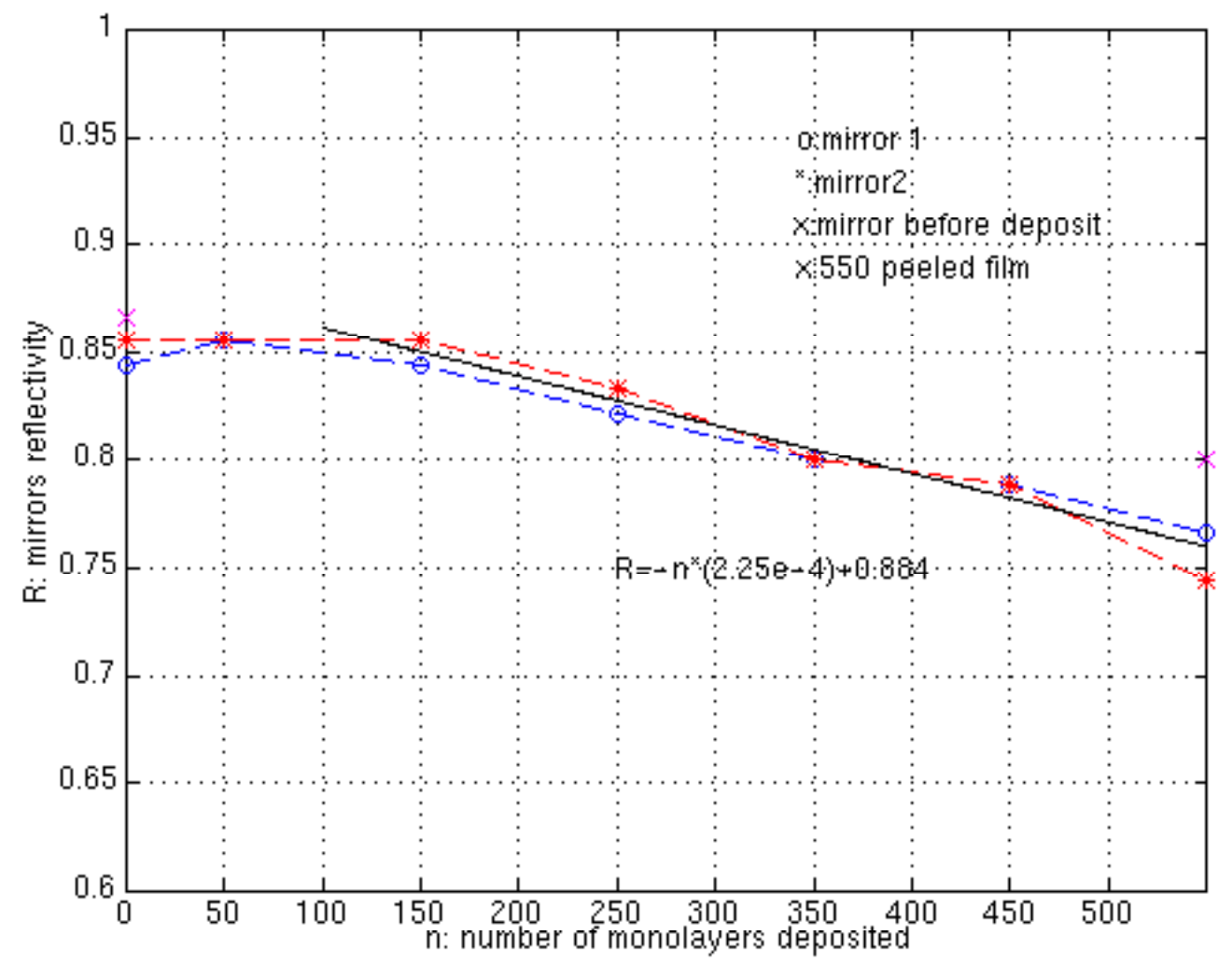

fig 5 : stainless steel mirrors reflectivity depending on the carbon thickness 
http://dx.doi.org/10.21611/qirt.2000.049

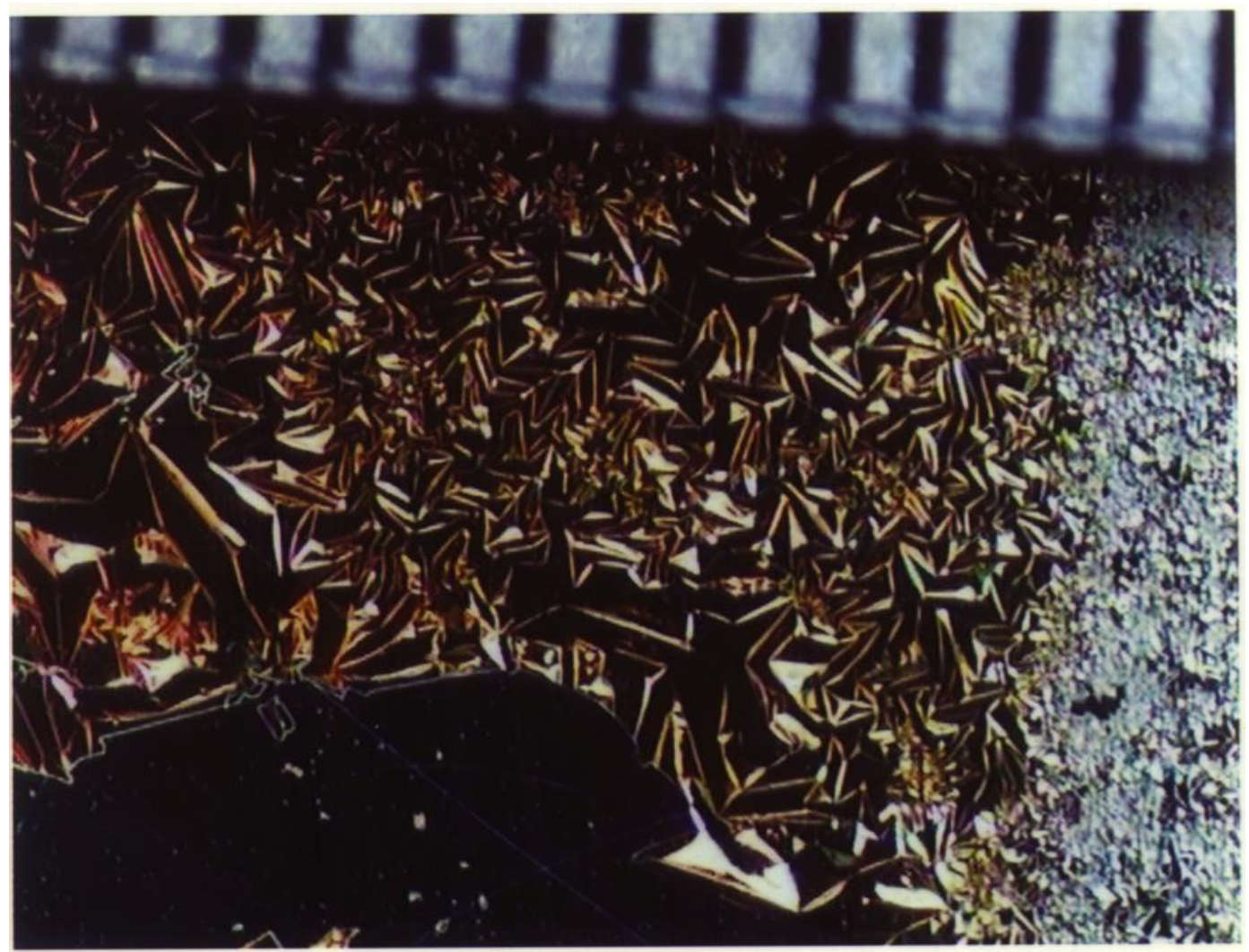

fig 6 : mirror macrophoto showing waves for the two thicker deposits 450 (right) and 550 (left) . on the upper part scale is shown : two black lines are separated by $1 \mathrm{~mm}$. 